\title{
ESO'S OPTICAL DETECTOR SYTEMS IN THE VLT OPERATIONS ERA
}

\author{
Dietrich Baade \\ European Southern Observatory, Karl-Schwarzschild-Str. 2, D-85748 Garching, Germany
}

\begin{abstract}
A brief review of the detector systems finished by ESO's Optical Detector Team (ODT) since 2000 is followed by a preview of forthcoming deliveries. Emerging trends are analyzed, and resulting actions and strategies are described.
\end{abstract}

Key words: CCDs, CCD controllers, detector systems

\section{INTRODUCTION}

The pre-2000 development work for and status quo of the CCD systems in use at ESO's La Silla and Paranal observatories were described by Beletic (2000). The single most important achievement were the design of the FIERA controller (Beletic, Gerdes, and DuVarney 1998), the PULPO house-keeping unit (Haddad and Sinclaire 1998), and the associated control software and their establishment as ESO's standard CCD control environment. By the year 2000, more than a dozen such systems had been deployed and were operating in Chile. Since then, 6 more systems were completed. After a few decades of total system operating time, it can now be safely said that, following the elimination of some initial dewar cleanliness problems, the reliability is excellent with downtimes of $0.5 \%$ or less. In fact, this performance poses a new kind of a challenge: the limited hands-on experience of the operations staff with trouble shooting is becoming a concern. 
The 6 new systems and the 3 ones currently in preparation are briefly described in Sect. 2 . On average, they are more complex and/or ambitious than the earlier ones, and the VLT instrumentation plan has been expanded following a call for proposals for $2^{\text {nd }}$ generation instruments. The associated trends are analyzed in Sect. 3, and Sect. 4 describes how ESO's Optical Detector Team (ODT) responds to these and other challenges.

The corresponding summary of ESO's work on IR detectors is provided by Finger (2002).

\section{RECENTLY DELIVERED AND FORTHCOMING CCD SYSTEMS}

The available space permits each system to be sketched only very crudely. The ODT Web site (home page: ww.eso.org/projects/odt) provides more in-depth information or, in some cases, will soon do so.

\subsection{Upgrades of FORS2 and EMMI (red arm)}

FORS2 and EMMI are multi-mode focal-reducer instruments at the VLT and the New Technology Telescope, respectively. They were initially equipped with one Tektronix TK2048 chip each with $2 \mathrm{Kx} 2 \mathrm{~K}$ 24- $\mu$ m pixels. In 2001 (FORS2) and 2002 (EMMI) these detectors were replaced with $2 \times 1$ mosaics of $2 \mathrm{Kx} 4 \mathrm{~K}$ MIT/LL CCID-20 high-resistivity devices with $15-\mu \mathrm{m}$ pixels. This increased the sky coverage by $50 \%$. However, at least as strong a driver was the much improved red sensitivity (see also Cavadore and Dorn 2000).

\subsection{Optical wavefront sensor for NAOS/CONICA}

The near-IR multimode instrument CONICA receives the telescope beam via the adaptive optics system NAOS, which features both an optical and an IR wavefront sensor. The former is described in detail by Feautrier et al. (2002); it is based on a Marconi CCD-55 detector. Other ODT work on wavefront sensor systems is presented by Dorn (2001) and Dorn et al. (2002). 


\subsection{Giraffe}

The high- and medium-resolution spectrograph Giraffe is a joint French/Suisse/ESO project. It can receive light through up to 130 fibers, which the FLAMES positioner can place anywhere in the 30arcmin field of view of the VLT (using 8 more fibers, FLAMES can simultaneously feed the UVES echelle spectrograph as well). The Giraffe detector system is built around one Marconi CCD44-82 chip $(2 \mathrm{~K} \times 4 \mathrm{~K}, 15-\mu \mathrm{m}$ pixels; for ODT test results for this type of device see Cavadore and Dorn 2000).

\subsection{VIMOS}

Built by a French/Italian consortium, this multi-object spectrograph amplifies the data-taking power of the VLT even more massively than Giraffe by supporting the collection of up to 750 spectra simultaneously, depending on spectral resolution. The field of view of about 22 arcmin diagonal is divided into 4 quadrants and optical trains, each of which is equipped with a separate detector head mounting one Marconi CCD4482 device. Two FIERA units control 2 detectors each.

\subsection{HARPS}

Late in 2002, the High-accuracy Radial Velocity Planetary Search Project will start its hunt for extra-solar planetary systems. It is being realized in a Suisse/French/ESO collaboration. The ODT-supplied detector system comprises a $2 \times 1$ mosaic of $2 \mathrm{Kx} 4 \mathrm{~K}$ chips with $15-\mu \mathrm{m}$ pixels (Marconi CCD44-82).

\subsection{MAD}

The Multi-conjugate Adaptive Optics Demonstrator will test enabling technology for ELTs in general and the OWL 100-m telescope in particular. It is based on Marconi CCD-50 split frame-transfer devices with $128 \times 128$ light-sensitive pixels, but only one quadrant each will be used. Up to 3 separate detector heads (out of a total of 5) need to be operated at frame rates up to several $100 \mathrm{~Hz}$ and with various synchronization patterns. A big challenge lies also in the combination of the specified low read noise with the requirements on weight, space, and positionability, which exclude LN2 cooling. Since delivery for system integration is foreseen for the end of Q1/2003, maximum use will be made of proven solutions adopted for the wavefront sensor of 
NAOS/CONICA. For a more comprehensive description see Cavadore, Marchetti, and Franza (2002).

\subsection{OmegaCAM}

In collaboration with the Osservatorio Astronomico di Capodimonte, ESO will erect the 2.6-m VLT Survey Telescope (VST) at the Paranal Observatory. The only instrument of the VST will be the UV-optimized wide-angle imager OmegaCAM, which is a joint German/Dutch/Italian/ESO enterprise. An $8 \times 4$ mosaic of $2 \mathrm{~K} \times 4 \mathrm{~K}$ Marconi CCD44-82 devices will fill the field of view (1.4 $4^{\circ}$ diagonal). Under the control of 2 FIERAs in a master/slave configuration, the total overhead per exposure will be 45 s (using one port per chip). A third FIERA will control 4 auxiliary CCDs of the same type, of which 2 will be employed for autoguiding and the other 2 for curvature wavefront sensing to enable the closed-loop operation of the telescope's active optics system.

\subsection{MUSE}

The Multi-unit Spectroscopic Explorer was proposed by a multinational consortium as a VLT second-generation instrument and recently selected for a Phase A study. It focuses on objects at redshifts, where their area density is so large that selective observations by means of positionable slits or fibers become very inefficient in sampling the required information. Therefore, the objective of MUSE is the complete stock taking over areas of 1 arcmin $x 1$ arcmin. Adaptive optics will enable a spatial sampling of 0.2 arcsec so that every observation will result in 90,000 spectra. The current optical design foresees to split the field into 24 beams, each of which would feature its own detector head with a $2 \mathrm{Kx} 4 \mathrm{~K} \mathrm{CCD}$. The detectors will need to have a red-optimized sensitivity to match the scientific high-z requirement.

\section{TRENDS}

There are strong tendencies towards a) fully paving the focal plane with silicon, b) ever more complex instruments with larger and larger wavelength coverage, and c) sensing of very weak and rapidly variable optical signals in (closed) control loops. FIERA has coped remarkably well with these challenges, which at the time of its conceptual design did not figure nearly as prominently as they do now. A concern though 
results from the weight, the volume, and the cooling requirements, which reach uncomfortable dimensions in the case of systems with many channels. The priceless advantage of being able to implement and operate all optical detector systems of ESO's observatories in one and only one environment (incl. a standard dewar) did, therefore, not need to be given up. However, continuous investments have been, and will also in future be, necessary to maintain the full power of this approach, which is integral part of the very successful VLT standardization strategy at large.

While there is undoubtedly a need to further reduce all possible nonphoton noise sources, especially for adaptive optics, scanning, and highresolution spectroscopy applications, there appears to be another, albeit speculative dimension which, if unfolded, would bear much bigger promise for astronomy at large: detectors with intrinsic wavelength resolution. A picture, that is, e.g., sometimes used in connection with adaptive optics, is the following: Suppose that a photon was emitted billions and billions of years ago, traveled over billions and billions of parsecs, hits M1, bounces off M2 - just to miss the slit. In imaging instruments, things are actually much worse: Chances are uncomfortably large that a filter discriminates against the impinging photon on account of its color although the detector could register it. This does not really appear scientifically or technically correct.

\section{ACTIONS AND INITIATIVES}

ESO's and the ODT's perception of the requirements to be met in the next couple of years have led to a number of activities, which in the following are briefly enumerated. For many of them, more comprehensive information is accessible from the ODT Web pages, the refurbishment of which is one of current ODT undertakings (www.eso.org/projects/odt).

Part of the FIERA concept is an LCU, which during the readout receives and orders the data. The initially utilized SPARC20 computer is no longer manufactured and will be replaced by an UltraSPARC, which necessitates the upgrade to a PCI bus (cf. Reyes Moreno et al. 2002). Studies to eventually move to a Linux box are underway.

The 36 CCDs of OmegaCAM require more temperature sensors and heaters than a current single PULPO can handle. PULPO is therefore 
being upgraded and will incorporate a small PC running Linux (see Reyes Moreno et al. 2002). The software will be ported to $C$.

OmegaCAM (and the contractual agreement with the supplier of the CCDs, which foresees that all 40+ devices are tested by ESO) was also the main driver for a comprehensive streamlining of both the control and the data reduction software of the ODT test bench (Amico and Böhm 1998), which now support the largely unsupervised acquisition and analysis of comprehensive data sets (cf. Christen et al. 2002). Discussions are ongoing of the benefits of converting the test bench to a fully VLT-like instrument, possibly with a VLT-like pipeline.

Following some unexpected problems, rigorous cleanliness standards were introduced for the CCD dewars, and numerous materials were studied for their suitability under vacuum and cryogenic conditions (cf. Deiries et al. 2002). Especially with a view toward the large OmegaCAM mosaic, a class-100 clean room was furnished.

The VLT control software (VCS) at large has reached a level of robustness and stability that permitted the length of release cycles to be increased from half a year to one year, thereby freeing capacities for development work and reducing the maintenance load on the operations staff. The FIERA software is integral part of the VCS and so followed this move.

ESO just recently placed a contract for the production of secondgeneration technical CCDs. Part of the responsibility for these systems now rests with the ODT, which thereby acquires a welcome direct contact with industrial systems.

In order to actively participate in the collection of experience with LLL CCDs, ESO and the Observatoire de Marseille have engaged in a joint evaluation project of a Marconi CCD65 device. First results are reported by Gach et al. (2002).

The usage of liquid nitrogen poses a permanent safety hazard to personnel and equipment (although careful safety standards have so far permitted any serious incident to be avoided at ESO) and requires significant operations and/or maintenance efforts. As a potential alternative, a Cryotiger ${ }^{\mathrm{TM}}$ system will be tested. 
As part of its strategic planning to be prepared for all future challenges, also with a view towards OWL readiness, ESO will conduct a study of the specific benefits that could be expected from a generic, wavelength-independent detector control and data acquisition environment. It will enable the ESO Management to decide whether to go ahead with such a project and, if yes, to establish cutting-edge, yet realistic requirements.

\section{ACKNOWLEDGEMENTS}

The author acknowledges that he has written the above paper. However, the actual work mentioned therein was performed by Andrea Balestra, Cyril Cavadore, Fabrice Christen, Claudio Cumani, Stéphane Darbon, Sebastian Deiries, Sandro D'Odorico, Reinhold Dorn, Christophe Dupuy, Jane Eskdale, Boris Gaillard, Christoph Geimer, Rolf Gerdes, Nicolas Haddad, Guy Hess, Joachim Hess, Evi Hummel, Olaf Iwert, Jean Louis Lizon, Robert Niemeczek, Gustavo Rahmer, Roland Reiss, Javier Reyes, Armin Silber, Peter Sinclaire, and others. Many of them also provided helpful comments on the manuscript.

The ESO participants in the SDW2002 workshop are grateful for the firework of Hawaiian hospitality and would be ready to host another workshop in the series; in any event: Auf Wiedersehen!

\section{REFERENCES}

Amico, P., Böhm, T. (1998): in Optical Detectors for Astronomy, eds. J.W. Beletic and P.

Amico, Astrophys. Space Sciences Lib., Vol. 228, Kluwer, Dordrecht, p. 95.

Beletic, J.W. (2000): in Optical Detectors for Astronom II, eds. P. Amico and J.W. Beletic,

Astrophys. Space Sciences Lib., Vol. 252, Kluwer, Dordrecht, p. 15

Beletic, J.W., Gerdes, R., DuVarney, R.C. (1998): in Optical Detectors for Astronomy, eds.

J.W. Beletic and P. Amico, Astrophys. Space Sciences Lib., Vol. 228, Kluwer, Dordrecht, p. 103

Cavadore, C., Dorn, R.J. (2000): in Optical Detectors for Astronom II, eds. P. Amico and

J.W. Beletic, Astrophys. Space Sciences Lib., Vol. 252, Kluwer, Dordrecht, p. 25 Cavadore, C., Cumani, C., Marchetti, E., Franza, F. (2002) : these proceedings 
Christen, F., Cavadore, C., Gaillard, B., Iwert, O., Kuijken, K., Baade, D., Darbon, S. (2002):

these proceedings

Deiries, S., Iwert, O., Cavadore, C., Geimer, Ch., Hummel, E. (2002): these proceedings

Dorn, R.J. (2001) : PhD thesis, Ruprecht-Karls-Universität, Heidelberg

Dorn, R.J., Burke, B.E., Beletic, J.M. (2002): these proceedings

Feautrier, Ph., Dorn, R.J., Rousset, G., Cavadore, C., Cumani, C., Hubin, N. (2002):

these

proceedings

Finger, G. (2002): these proceedings

Gach, J.L., Guillaume, C., Cavadore, C., Boissin, O. (2002): these proceedings

Haddad, N., Sinclaire, P. (1998): in Optical Detectors for Astronomy, eds. J.W. Beletic and $P$.

Amico, Astrophys. Space Sciences Lib., Vol. 228, Kluwer, Dordrecht, p. 131

Reyes Moreno, J., Balestra, A., Geimer, C., Haddad, N. (2002): these proceedings 